\title{
Tunable grating with active feedback
}



Proc. SPIE 8687, Electroactive Polymer Actuators and Devices (EAPAD) 2013, 86872F (April 9, 2013); doi:10.1117/12.2009358

Copyright 2013 Society of Photo-Optical Instrumentation Engineers. One print or electronic copy may be made for personal use only. Systematic electronic or print reproduction and distribution, duplication of any material in this paper for a fee or for commercial purposes, or modification of the content of the paper are prohibited.

http://dx.doi.org/10.1117/12.2009358 


\title{
Tunable grating with active feedback
}

\author{
Samuel Rosset ${ }^{a}$, Benjamin M. O'Brien ${ }^{b}$, Todd Gisby ${ }^{b}$, Daniel Xu ${ }^{b}$, Herbert R. Shea ${ }^{a}$ and Iain \\ A. Anderson ${ }^{b}$ \\ ${ }^{a}$ Ecole polytechnique fédérale de Lausanne, Lausanne, Switzerland; \\ ${ }^{b}$ Biomimetics Laboratory, Auckland Bioengineering Institute, University of Auckland, \\ New-Zealand
}

\begin{abstract}
We report on the use of capacitive self-sensing to operate a DEA-based tunable grating in closed-loop mode. Due to their large strain capabilities, DEAs are key candidates for tunable optics applications. However, the viscoelasticity of elastomers is detrimental for applications that require long-term stability, such as tunable gratings and lenses. We show that capacitive sensing of the electrode strain can be used to suppress the strain drift and increase the response speed of silicone-based actuators. On the other hand, VHB actuators exhibit a time-dependent permittivity, which causes a drift between the device capacitance and its strain.
\end{abstract}

Keywords: Silicone, response speed, metal ion implantation, conductive rubber, carbon grease

\section{INTRODUCTION}

Dielectric Elastomer Actuators (DEAs) are known for their large strain compared to other actuation technologies. Many applications which directly benefit from the large strain and intrinsic compliance of DEAs have been developed: robotic manipulators, ${ }^{1-3}$ Micropumps, ${ }^{4}$ haptic feedback devices,${ }^{5}$ stretchers of biological cells, ${ }^{6}$ and tunable optics ${ }^{7-9}$ to name a few. The field of tunable optics is particularly challenging, because most of the applications require precise positioning, and the ability to keep a stable position for a given amount of time. DEAs are interesting actuator candidates for this field, because they can provide a much larger tuning range compared to standard MEMS approaches. For example silicon-based tunable gratings have a typical tuning range of a few percent, ${ }^{10,11}$ whereas DEA-based solutions provide strains larger than $10 \%$ (we have obtained up to $27 \%$ change in grating period). However, because of the inherent viscoelastic behaviour of these actuators, DEA-based optical devices present a slow response speed and their strain keep increasing with time, preventing them to hold a stable position. Despite their very large tuning range, this makes DEAs unsuitable for most real-world optical application requiring precision.

The most widely used dielectric for DEAs is the 3M VHB adhesive tape, mainly because of the high strain it can produce, and because it is readily available in thin-film form. However, this material is extremely viscoelastic as shown on figure 1. For this particular case, the actuator was made from a VHB 4905 membrane equi-biaxially prestretched to 9 times its initial area and had carbon grease electrodes (Nyogel $756 \mathrm{G}$ ). The device needs 163 seconds to reach $90 \%$ of its equilibrium strain. The figure also shows the step response of a similar actuator made with a silicone membrane (RS 692-542, prestretched to 1.5 times its initial area and with carbon grease electrodes), which reacts much faster and needs only 3 seconds to reach $90 \%$ of its final strain. As we have shown in a previous study, the response speed of DEA do not only depend on the dielectric material and its prestretch, but also on the electrode material. ${ }^{12}$

One possible approach to increase the response speed of DEAs and stabilise their output to a controlled value is to use active feedback: the strain is monitored and the applied voltage actively regulated in order to keep it at the desired value. The traditional approach to closed-loop operation is to have an external sensor measuring the process variable (for example the actuator strain) and comparing it with a desired set-point. A controller computes the voltage to apply, based on the value of the error between the actual value and the set-point (and possibly also the time history of this error and its rate of change). This requires the use of an external sensor to measure the value of the process variable, which increases the complexity and cost of the device and contradicts one of the main arguments in favour of plastic-based actuators: the low-cost approach.

contact author: samuel.rosset@a3.epfl.ch

Electroactive Polymer Actuators and Devices (EAPAD) 2013, edited by Yoseph Bar-Cohen,

Proc. of SPIE Vol. 8687, 86872F · (c) 2013 SPIE · CCC code: 0277-786X/13/\$18 · doi: 10.1117/12.2009358

Proc. of SPIE Vol. $868786872 \mathrm{~F}-1$ 


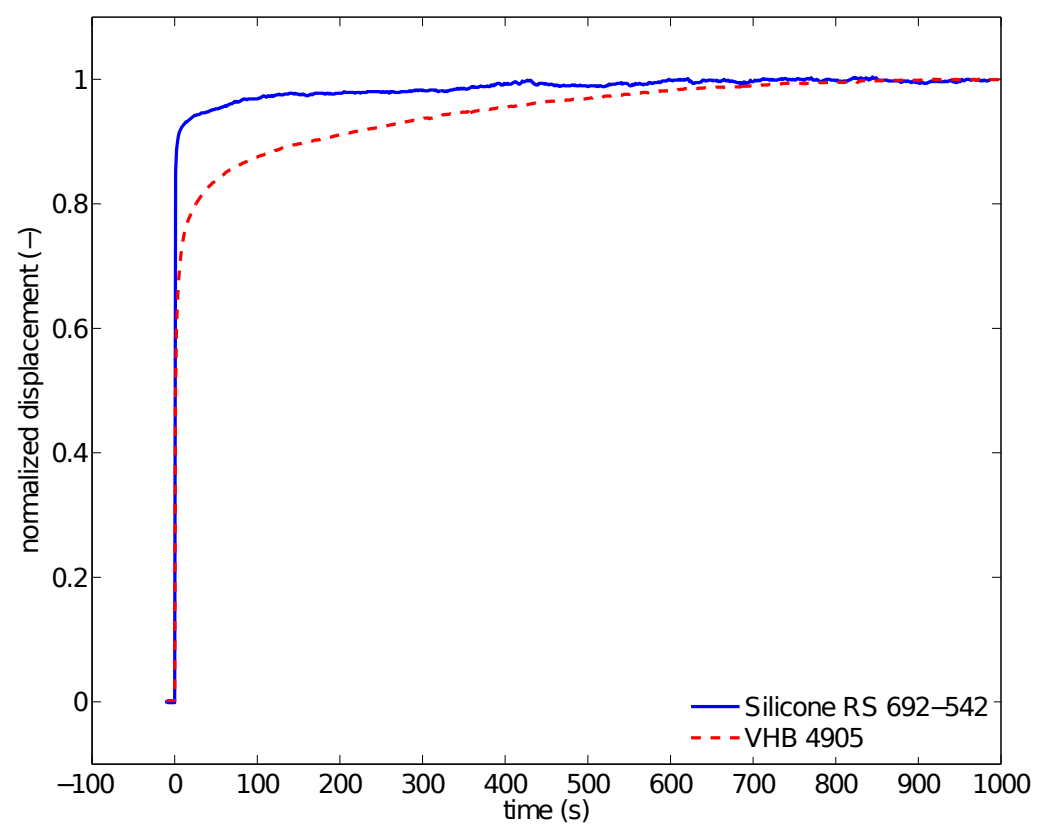

Figure 1. Normalized strain response to a voltage step input for a silicone membrane (RS 692-542) and a 3M VHB membrane (VHB 4905).
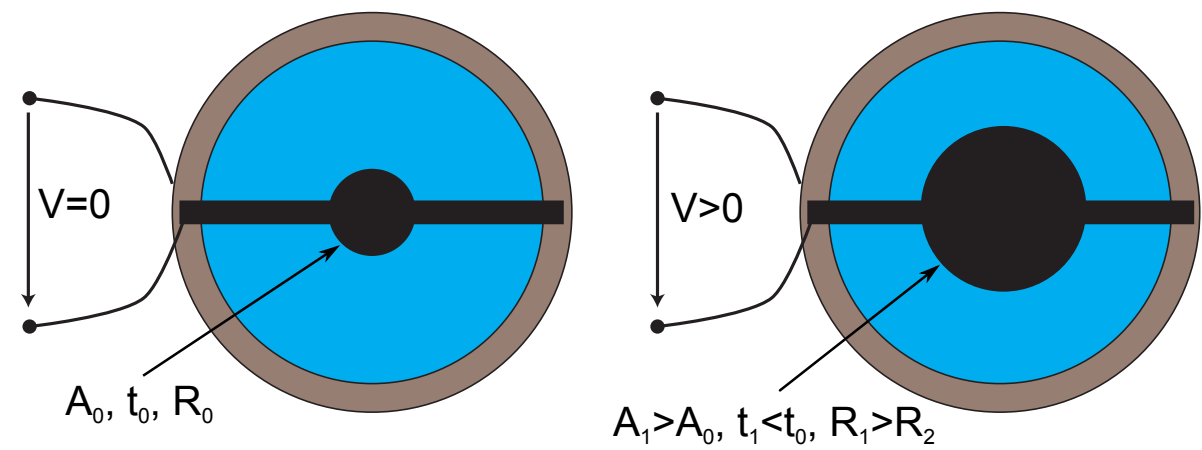

Figure 2. Circular actuator on a prestretched membrane. The application of a voltage causes the electrode to expand in plane. This leads to an increase of the electrode area and resistance and a decrease of the dielectric thickness in the electrode zone.

\subsection{Self-sensing for active feedback control}

Instead of the classical approach to feedback control, which clearly separates the actuator from the sensor monitoring the displacement of the former, we can treat the DEA device not as a simple actuator, but as a smart device not only able to move, but also to sense its own strain through the use of self-sensing. When a DEA is activated, its electrodes expand, causing an increase in their area, a decrease of the dielectric thickness in the electrode zone, and an increase of the series resistance of the electrode (figure 2). These parameters can be used to estimate the strain of an actuator through self-sensing.

The first approach, resistive self-sensing, consists in using the change of resistance of the electrodes as a measurement of strain. This can be done by applying a small voltage difference between the two legs of the ground electrode and measuring the differential current, as presented in one of our previous publication. ${ }^{13}$ However, resistive self-sensing is quite sensitive to the type of electrode, with some of them exhibiting an important hysteresis or bumps when the strain rate changes, making the estimation of the actuator strain through the resistance of its electrode difficult. ${ }^{13}$

The second approach consists in tracking modification of the device geometry (increase of the electrode surface and decrease of the dielectric thickness) through the measurement of the capacitance of the device, also known as capacitive self-sensing. ${ }^{14-16}$ Unlike resistive sensing which depends on many different parameters in addition 


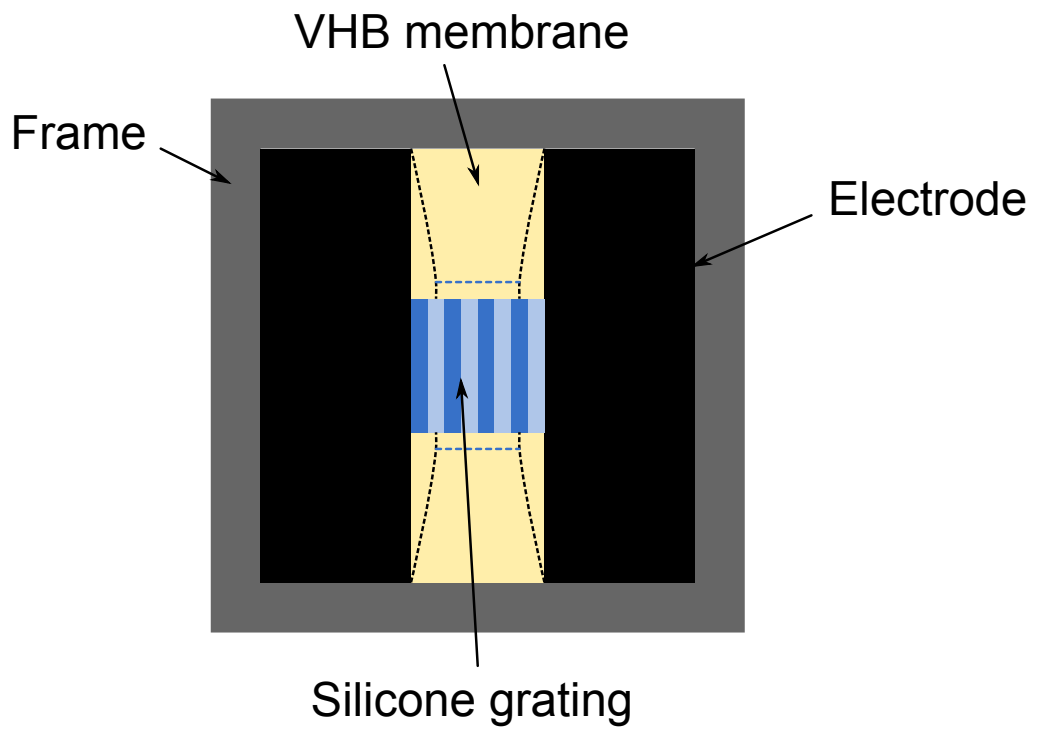

Figure 3. Tunable grating. When a voltage is applied to the electrodes, their expansion compresses the grating, which changes its spacial frequency. The drawing illustrates the device at rest state, with the dashed lines indicating the deformation when activated.

to the strain, the value of the capacitance is only dependent on the electrode strain, membrane compression and relative permittivity of the membrane. The thickness compression and electrode expansion are linked by the incompressibility of the material, and the relative permittivity is assumed to be a constant in the strain range that can be electrically induced. (The relative permittivity of VHB has been shown to decrease when the material is prestretched. ${ }^{17}$ However, VHB membranes are typically prestretched to more than 3 times their initial size, whereas the electrically-induced strain we are considering here lies between $10 \%$ and $20 \%$ ). There is therefore an injective function linking the strain to the capacitance, and the capacitance can thus be used as a proxy for the device deformation, if the function $\lambda=f(C)$ is known, with $\lambda$ the stretch, and $C$ the capacitance of the device.

In the case of the simple circular actuator shown on figure 2 , if $r_{0}$ and $t_{0}$ represent the initial radius of the electrode and thickness of the dielectric, $\lambda_{r}$ the radial stretch $\left(r=r_{0} \lambda_{r}\right)$, and $\lambda_{t}$ the thickness stretch $t=t_{0} \lambda_{t}$, the capacitance $C$ of the device can be expressed as:

$$
C=\frac{\epsilon \pi r^{2}}{t}=\frac{\epsilon \pi r_{0}^{2} \lambda_{r}^{2}}{t_{0} \lambda_{t}}=C_{0} \frac{\lambda_{r}^{2}}{\lambda_{t}}
$$

Additionally, taking into account the incompressibility of elastomers $\left(\lambda_{r}^{2} \lambda_{t}=1\right)$, equation 1 can be rewritten as:

$$
C=C_{0} \frac{\lambda_{r}^{2}}{\lambda_{r}^{-2}}=C_{0} \lambda_{r}^{4},
$$

which shows that the capacitance is only dependent on the radial stretch, and on the initial capacitance of the device $C_{0}$. Depending on the actuator configuration, and particularly on the boundary conditions, the equation 2 is modified, but the capacitance is generally linked to the stretch via a power relation $C=C_{0} \lambda_{r}^{n}$.

\subsection{Tunable grating actuator}

A tunable grating based on a DEA actuator with a soft and deformable grating structure has been chosen for this work. This is indeed a good illustration of a tunable optical device which can take full advantage of the large tuning range of DEAs, but whose deformation must be accurately controlled and stable in time to be of any use. The geometry is based on the design by M. Kollosche et al. ${ }^{9}$ and consists of a VHB 4905 membrane equi-biaxially prestretched to 9 times its original area and fixed on a square frame. Carbon grease electrodes 


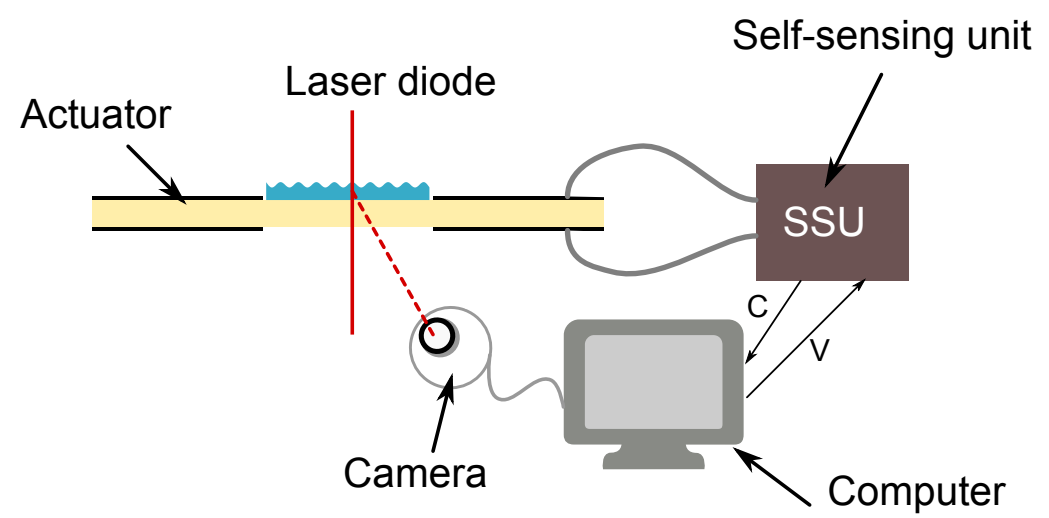

Figure 4. Experimental setup: the actuator is connected to an electronic self-sensing unit (SSU) capable to drive the actuator while reading the capacitance. The SSU sends the capacitance value to the computer, which calculates the driving voltage and sends it to the SSU. A camera is used to track the position of the first order diffraction spot of a Laser diode shone through the grating.

(Nyogel 756G) were applied on two sides of the membrane, and a soft deformable grating replicated into a thin silicone membrane was placed at the center (figure 3).

When a voltage is applied to the device, the electrode expansion compresses the soft grating. The spacial frequency of the grating structure increases, which increases the diffraction angle. The gratings used for this study have a spatial frequency of 500 lines $/ \mathrm{mm}$.

\subsection{Experimental setup}

The tunable grating was driven by an electronic self-sensing unit (SSU) developed at the Biomimetics laboratory. The unit is capable of applying a voltage up to $5 \mathrm{kV}$ to a DEA, while monitoring the value of its capacitance with a $1 \mathrm{pF}$ resolution. The SSU has a USB port, which allows data exchange with a computer. For the test setup (figure 4), the SSU was connected to the actuator, and linked via USB to a computer. A simple PI (proportionalIntegral) controller was implemented with Labview: The SSU measures the capacitance value of the DEA, which is transmitted to the controller, where it is compared to the desires capacitance set point. A driving voltage is computed, depending on the difference between the actual capacitance and the set-point and time-history thereof. The voltage value is transmitted to the SSU, which applies it to the actuator. The objective is to keep the grating period to a constant value for an extended period of time via regulation of the device capacitance to a constant value. To evaluate the effectiveness of the controller based on capacitive self-sensing, a laser diode $(\lambda=670 \mathrm{~nm})$ is shone through the grating and the position of the first order diffraction spot is monitored with a camera. The camera is used for verification only and is not part of the feedback loop.

\section{RESULTS}

As a first validation of the method, the relation between the capacitance and the deformation must be evaluated (figure 5). A ramping voltage between 0 and $3000 \mathrm{~V}$ was applied to a device, and its capacitance and the stretch of the electrode was measured. The test was conducted 4 times and showed a good repeatability. A power fit leads to the relation $C=910 \lambda^{2.41} \mathrm{pF}$. The quantification of the stretch value is due to the $50 \mu \mathrm{m}$ resolution of the laser sensor used to measure the strain. From this measurement, one sees that the electrode strain can be defined by the capacitance of the device. For example, an electrode stretch of 1.05 corresponds to a capacitance value of $1023 \mathrm{pF}$. The stretch of the grating, which is what we ultimately want to control, is linked to the electrode stretch by the following geometrical constraint which state that the sum of the length of the two electrodes plus that of the grating is a constant:

$$
2 \lambda_{e} l_{e}+\lambda_{g} l_{g}=2 l_{e}+l_{g} \Rightarrow \lambda_{g}=2\left(1-\lambda_{e}\right) \frac{l_{e}}{l_{g}}+1,
$$




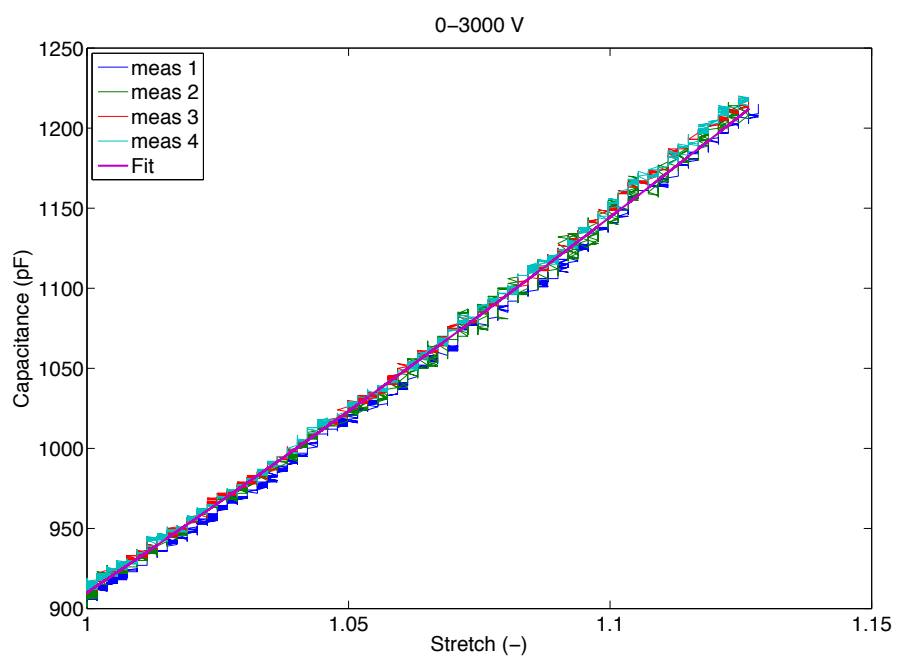

Figure 5. Capacitance as a function of electrode stretch for an applied voltage between 0 and $3 \mathrm{kV}$. The measurement is repeated 4 times and shows a good repeatability. A power curve fit leads to $C=910 \lambda^{2.41} \mathrm{pF}$



Figure 6. Closed loop operation of the tunable grating with a PI controller. The capacitance setpoint is changed from $580 \mathrm{pF}$ to $800 \mathrm{pF}$ at $t=0$. Top: Voltage applied to the device, center: capacitance of the device, bottom: grating period.

where $\lambda_{g}$ and $\lambda_{e}$ are respectively the stretch of the grating and of the electrodes; $l_{e}$ and $l_{g}$ the initial length of the electrode and of the grating. The factor 2 comes from the fact that there are two electrodes of equal size on each side of the grating.

To test the tunable grating, an actuator was connected to the setup described in paragraph 1.3 and figure 4 . We used a standard PI controller with a proportional gain $K_{p}=5$ and an integral gain $K_{i}=12$. The set-point is initially set to $580 \mathrm{pF}$ and suddenly increased to $800 \mathrm{pF}$ to study the reaction of the actuator-controller system to a brutal change of set point. The values of the voltage applied to the actuator, and the capacitance read by the SSU were saved. In parallel, the displacement of the first order diffraction spot is tracked by a camera, and the corresponding grating period calculated. The data from the camera is used to check whether the system is working as expected: even though the capacitance of the device is the process variable which can be measured and regulated by the driving electronics, it is the grating period which we want to control and stabilize. Figure 6 shows the values of the applied voltage (top), the capacitance (center), and the grating period (bottom) for 

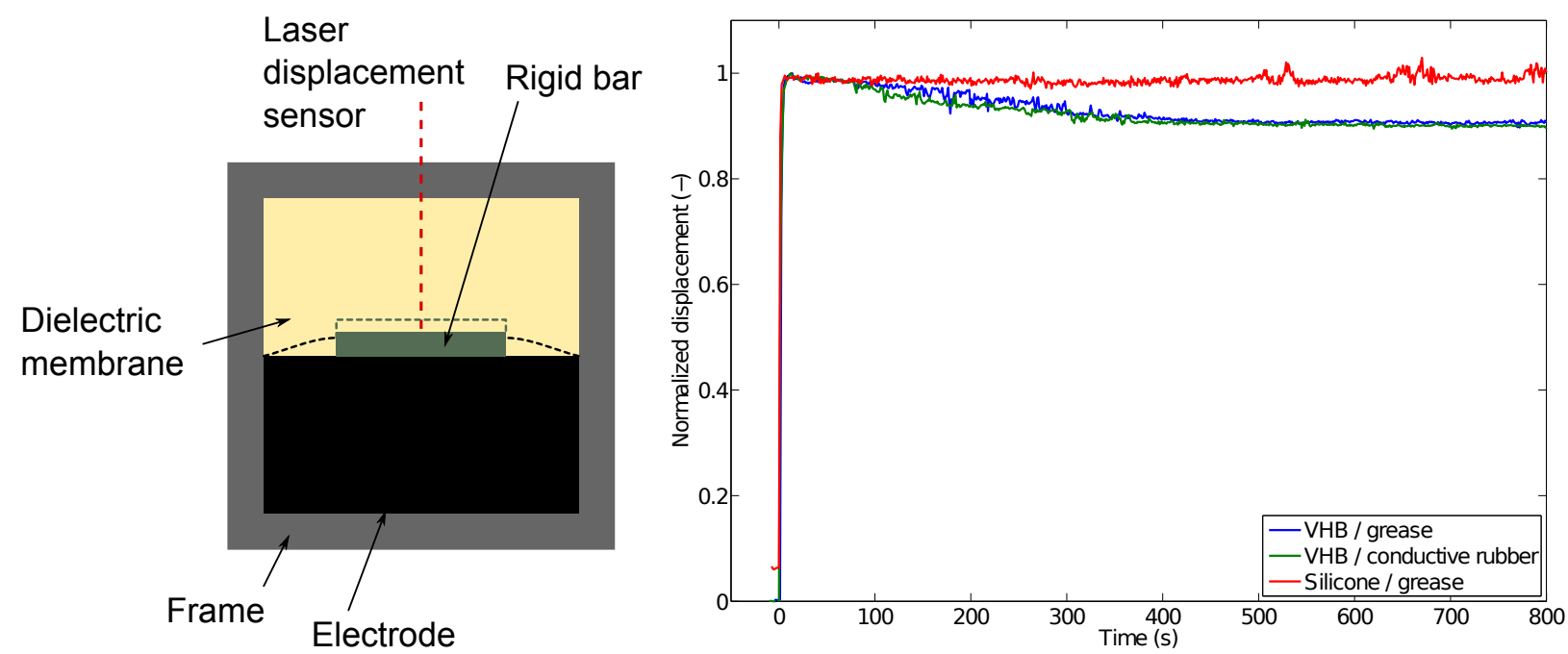

Figure 7. Left: test actuator to investigate the drift between the strain and the capacitance. The electrode strain causes the rigid bar to move and its displacement is recorded with a laser displacement sensor. Right, normalized displacement obtained for closed-loop operation in the case of a VHB membrane with carbon grease electrodes and conductive rubber electrodes, and for a silicone membrane with carbon grease electrodes. The capacitance is kept constant by the controller for $t>0$.

60 seconds after the change of capacitance set-point. From the controller/SSU perspective, the system behaves exactly as expected, with the capacitance quickly changing from $580 \mathrm{pF}$ to $800 \mathrm{pF}$ (rise time: $1.45 \mathrm{~s}$ ) and staying at the final value: much faster and much more stable than open-loop operation. The capacitance is kept constant by a gradual decrease of the driving voltage to compensate for the viscoelastic creep of the device. The grating is compressed and its period decreases from $2.01 \mu \mathrm{m}$ to $1.75 \mu \mathrm{m}$. But instead of remaining to this value, the period then slowly increases, and reaches $1.77 \mu \mathrm{m}$ after 60 seconds, even though the capacitance of the device doesn't change. This slow increase of the strain at a constant capacitance value continues for about 5 minutes before stabilizing, at which time the displacement drift represents 8-10\% of the step height depending on devices. It was consequently impossible to keep the grating at a precise stretch value by controlling its capacitance.

This drift behaviour between capacitance and strain must be investigated more closely, as it represents an important obstacle to the use of capacitance self-sensing for closed-loop operation. Because of the numerous steps linking the voltage applied to the electrode to the computation of the grating period, each of them possibly bringing some error, a simpler geometry was chosen to verify the existence of a injective relation between the strain of a device and its capacitance. The modified actuator (figure 7 left) consists of the same basic geometry than the grating, but a single electrode is used, and a rigid bar is placed on the membrane along the electrode. Upon application of a voltage, the bar moves, and its displacement is recorded with a laser displacement sensor. Three different actuators were built and tested: 1) a VHB membrane with carbon grease electrodes (the same configuration as used for the grating. 2) a VHB membrane with carbon rubber electrodes (carbon black mixed into a silicone matrix) to verify if the drifting behaviour is due to the electrodes: the grease might diffuse into the membrane and change the dielectric constant of VHB, whereas we expect the silicone/carbon black mix to be much more stable. 3) An actuator with a silicone membrane (RS 692-542) and carbon grease electrodes to study the impact of the dielectric membrane material. The three actuators were driven in closed loop mode for 800 seconds at a capacitance value corresponding to a driving voltage of $2.2-2.5 \mathrm{kV}$ (figure 7 right). The results show that regardless of the electrode type, the actuators with a VHB dielectric membrane present a decrease of about $10 \%$ of the displacement step during the first 5 minutes of the test before stabilizing to the final value. With the silicone membrane however, the displacement stays constant when the capacitance is kept at a constant value. 


\section{DISCUSSION}

The results of the above tests shows that the drift between the capacitance and the strain of the grating observed on figure 6 is not due to the device structure itself, but to the VHB membrane, as it can be systematically reproduced on different actuator geometries based on this membrane material. The influence of the grease electrodes, which can diffuse in-plane or into the membrane, can also be eliminated because an actuator made with carbon black particles in a crosslinked elastomeric matrix showed the same behaviour. This might be due to different causes, such as a relative permittivity changing with time under the influence of a high applied electric field. When we replaced the VHB membrane by a silicone membrane the problem disappeared and it was therefore possible to keep the strain of silicone-based actuators constant by regulating the capacitance of the device to a constant value.

It can therefore be concluded that the use of capacitive self-sensing with a VHB-based DEA is not straightforward, particularly if a positioning precision better than $10 \%$ is required. However, it can still be advantageously used to speed up the response speed of actuator by tuning the gain of the controller. The difficulty to control the output strain of VHB actuators is unfortunate, as this material would benefit the most from closed-loop operation because of its high viscoelasticity. Closed-loop operation of VHB actuators is of course possible using the standard approach to active feedback through the use of an external sensor, without using the capacitance of the device. On the other hand, silicone elastomer (at least RS 692-542 from RS components) do not suffer from this drifting behaviour and are therefore well-suited for capacitive self-sensing.

\subsection{Regulator optimization}

Standard PID (or any variation thereof, such as PI) controllers are not well suited to drive DEAs due to the quadratic relationship between Voltage and strain. This causes the properties of the controller (response time, steady-state error, stability, noise, etc.) to be dependent on the setpoint of the controller. A noise in the capacitance reading $(\Delta C)$ for a $\mathrm{P}$ regulator leads to a noise $\Delta V=K_{p} \Delta C$ in the voltage output. If the actuator is in a low strain (i.e. low voltage) state, the noise in the driving voltage is much less noticeable than for an actuator which is at a high strain level $\epsilon$, because $d \epsilon / d V$ increases with the voltage $\left(\epsilon \propto V^{2}\right)$.

To better understand how a DEA behaves when it is driven by a standard PID controller, and to find a better alternative, we have modelled a DEA in closed-loop mode with Matlab Simulink and have experimented with different regulator settings. We first characterized the capacitance as a function of applied voltage of a device by applying a slow voltage ramp between 0 and $3000 \mathrm{~V}$. A polynomial function of the form

$$
C_{\infty}=C_{0}+\alpha V^{2}+\beta V^{4}
$$

was fitted to the data points, where $C_{\infty}$ represents the steady state capacitance, $C_{0}$ the initial capacitance of the device, $V$ the applied voltage, and $\alpha$ and $\beta$ two constants obtained from the curve fitting. The time dependence of the capacitance was modelled by applying a voltage step to an actuator and measuring the capacitance as a function of time, which was then fitted to a Prony series in the form:

$$
C(t)=C_{0}+\Delta C \sum_{i=1}^{n} a_{i}\left(1-e^{-t / b_{i}}\right) .
$$

From our experimental data a sum of three exponentials $(n=3)$, each with its respective time constant, was seen to closely fit the data. Finally, by grouping the two equations together, one can express the dependence of the capacitance on both time and applied voltage by:

$$
C(V, t)=C_{0}+\left(\alpha V^{2}+\beta V^{4}\right)\left[\sum_{i=1}^{n} a_{i}\left(1-e^{-t / b_{i}}\right)\right]
$$

To test the validity of the model, the actuator was driven in open-loop with three consecutive voltage steps: form $0 \mathrm{~V}$ to $1 \mathrm{kV}$ at $t=2 \mathrm{~s}$, then to $2 \mathrm{kV}$ at $t=4 \mathrm{~s}$ and finally to $3 \mathrm{kV}$ at $t=6 \mathrm{~s}$ until $t=10 \mathrm{~s}$. The capacitance was measured using the SSU, and the the theoretical behaviour calculated using equation 6 . The 

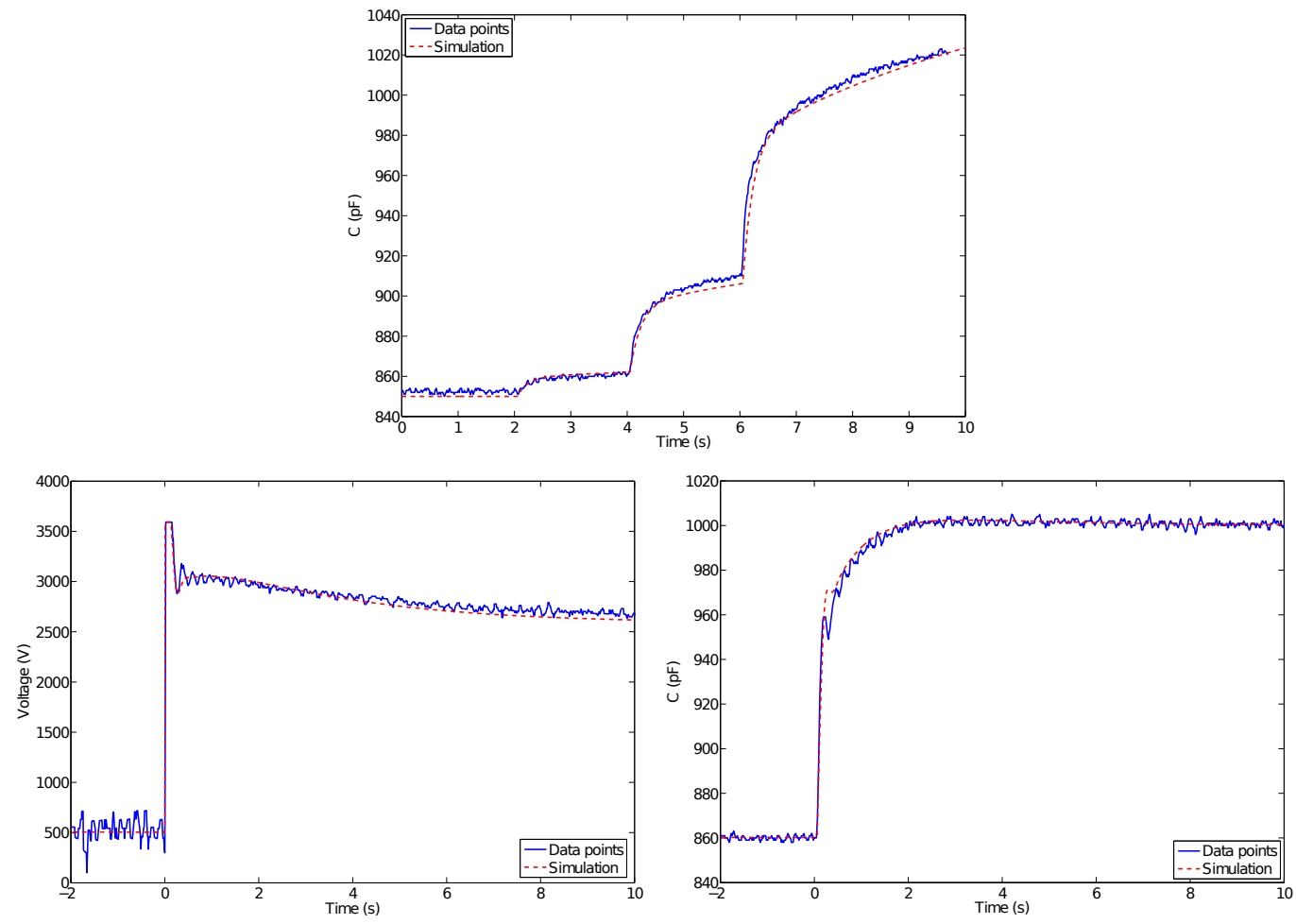

Figure 8. Top: Open loop test with three consecutive voltage steps: $1 \mathrm{kV}, 2 \mathrm{kV}$ and $3 \mathrm{kV}$. Comparison with an actuator and its model. Bottom: measured data and model in the case of closed loop operation with a PI regulator for a capacitance set-point of $1000 \mathrm{pF}$.
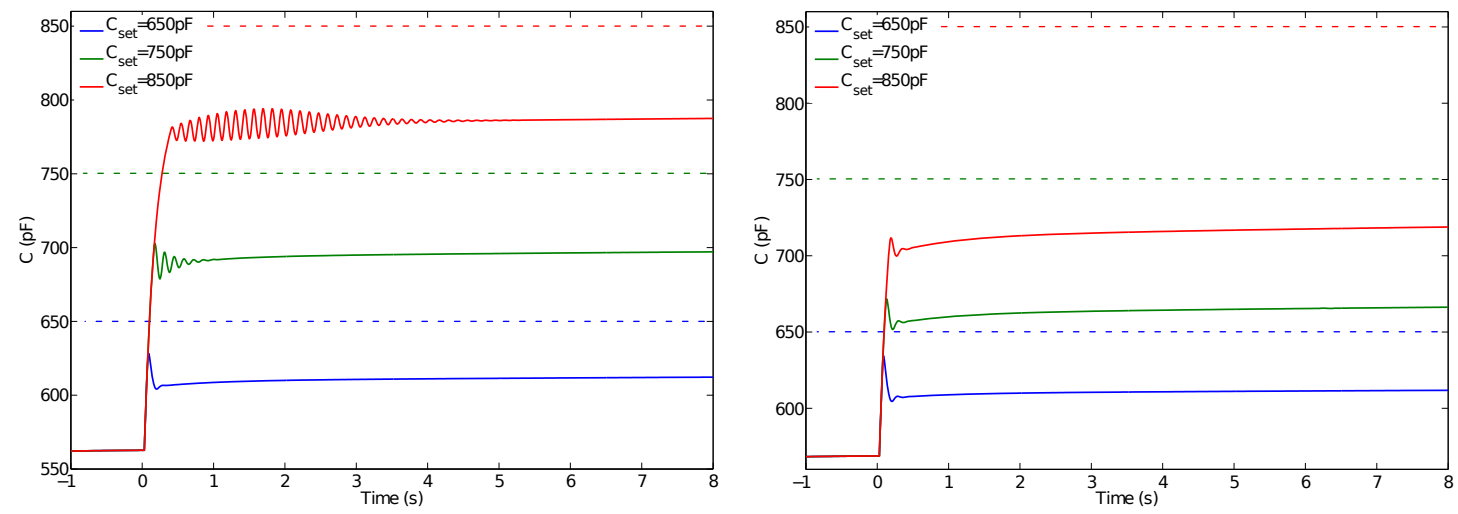

Figure 9. Left: typical response of an actuator driven by a simple P controller for different capacitance setpoints. Right: response for a $\mathrm{P}$ regulator with a variable gain to compensate for the quadratic relationship between voltage and strain. The dashed lines represent the set points.

unknown parameters of the model were obtained before the test, using a voltage ramp test and a voltage step test as described above. The measured capacitance is plotted on figure 8 top, alongside the model, which is in excellent accordance with the data. To further verify if the numerical model can be used to predict closed loop operation, the actuator was then tested when driven by a PI controller with a set-point change from $800 \mathrm{pF}$ to $1000 \mathrm{pF}$. In parallel, the controller was added to the Matlab Simulink model with the same sampling frequency. The measured data and predicted values are shown on figure 8 bottom, for the driving voltage (left) and capacitance (right). The model can correctly predict the behaviour of the actuator driven in closed-loop mode and can consequently be used to optimize the control scheme. 

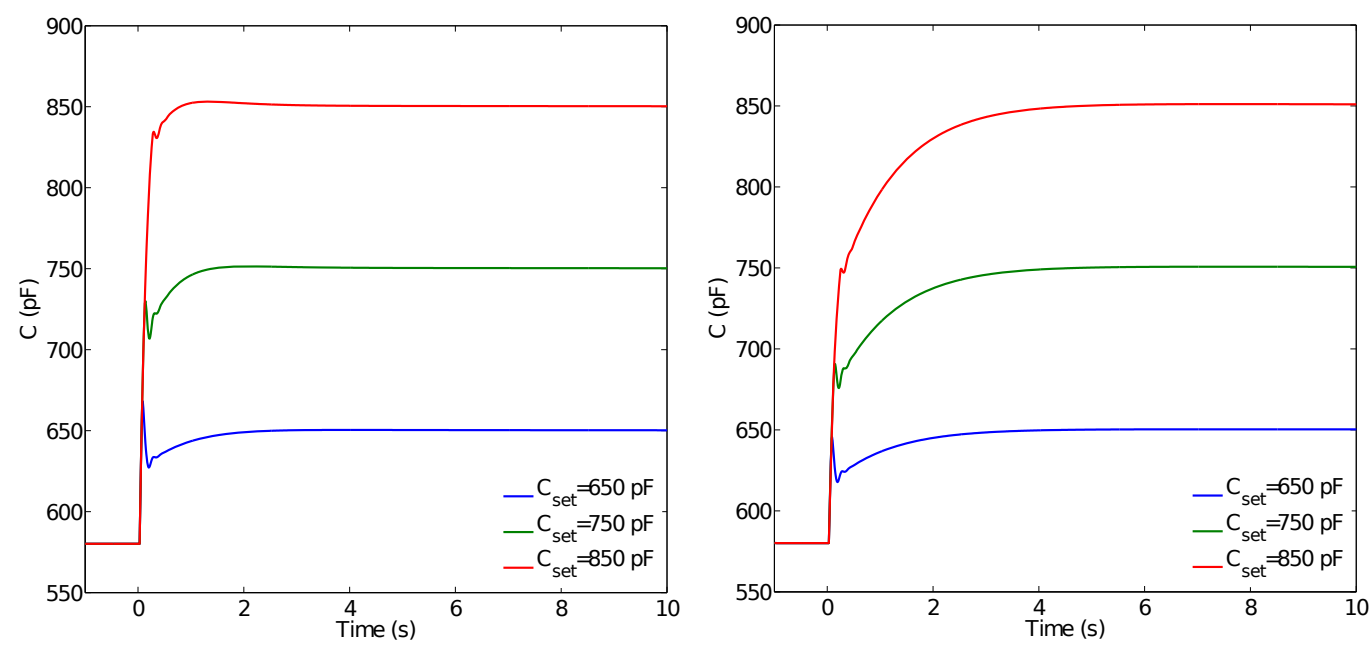

Figure 10. Left: PI regulator with a the variable proportional gain and a fixed integral gain $K_{i}=60$. Right: both the proportional and integral gain are scaled by the variable correction factor.

Figure 9 left shows the typical expected response of a DEA driven by a simple $\mathrm{P}$ regulator (in this particular example with a gain of 50) for a jump from $C_{0}$ to three different capacitance set-points. The dynamics of the response clearly depend on the set-point value, which is highly undesirable. It is for example obvious that the system becomes less stable and presents more oscillations when the target capacitance is increased In addition, the relative steady-state error $\epsilon$ compared to the difference between $C_{0}$ and the set-point (equation 7 ) is also dependent on the set-point.

$$
\epsilon=\frac{C_{\infty}-C_{s e t}}{C_{\text {set }}-C_{0}}
$$

with the indices $\infty$, set, and 0 referring respectively to the steady-state capacitance, the set-point, and the initial capacitance before the jump. For the particular case presented on figure 9 left, the relative errors for the different set-points are given in the $2^{\text {nd }}$ column of table 1 .

Table 1. Relative steady-state error for the different set-points for a $\mathrm{P}$ regulator with a fixed gain ( $2^{\text {nd }}$ column), and a modulated gain ( $3^{\text {rd }}$ column).

\begin{tabular}{|c|c|c|}
\hline$C_{\text {set }}(\mathrm{pF})$ & $\epsilon$ (\%) fixed gain & $\epsilon(\%)$ variable gain \\
\hline 650 & -39 & -39 \\
\hline 750 & -26 & -42 \\
\hline 850 & -20 & -43 \\
\hline
\end{tabular}

To obtain a dynamic behaviour which is independent from the set-point, we multiplied the fixed gain $K_{p}$ of the regulator by a variable gain depending on the sensitivity of the capacitance to the applied voltage at the current actuator state:

$$
K_{p}^{\prime}=\left.K_{p} \frac{d V}{d C}\right|_{C=C(t)}
$$

The simulated responses with the variable gain are shown on figure 9 right. The growing instability with increasing target values has been suppressed, and the relative steady state error presents much less variation (table $1,3^{\text {rd }}$ column).

To suppress the steady-state error, it is necessary to add an integrator to the controller (PI controller). Two cases are calculated: 1) the proportional part of the controller uses the variable gain defined by equation 8 and the integral part uses a fixed gain $K_{i}=60$ and 2) the variable correction factor $d V / d C$ is applied to both the proportional and integral gains. The simulated capacitance responses for the three different set-points are shown on figure 10. The main influence of the variable integral gain concerns the response speed of the system: 
when the $K_{i}$ is fixed, the rise time depends on the set-point value, with a faster response for higher capacitance setpoint, whereas when the integral gain is also modulated by the sensitivity of the capacitance variation to the applied voltage, the response-time is much less sensitive to the set-point value, as reported in table 2 .

Table 2. Rise time $t_{r}$ for different capacitance set-points

\begin{tabular}{|c|c|c|}
\hline$C_{\text {set }}(\mathrm{pF})$ & $t_{r}(s)$ fixed $K_{i}$ & $t_{r}(s)$ variable $K_{i}$ \\
\hline 650 & 0.96 & 1.67 \\
\hline 750 & 0.55 & 1.70 \\
\hline 850 & 0.26 & 1.71 \\
\hline
\end{tabular}

\section{CONCLUSIONS}

In this work, we have shown that capacitive self-sensing is an elegant way to implement closed-loop operation for DEAs, because it doesn't require additional sensing elements external to the actuators, nor additional wires. The technique is based on the fact that DEAs are smart devices which can be used concurrently as actuators and sensors. Through the example of a tunable grating based on a VHB membrane, we have demonstrated that closed-loop operation through capacitive self-sensing allows to increase the response speed of the actuators, but does not permit a precise control of the actuation position, due to a drift between the capacitance of the device and its strain. This drift has been attributed the VHB membrane, as silicone-actuators could be stabilized at a constant strain when driven in closed-loop. The combination of silicone membranes with capacitive self-sensing for closed-loop operation allows to obtain fast and precise DE actuators and opens the way to a broad range of new applications which require a precise position control, in addition to the large strain which characterizes DEAs.

\section{ACKNOWLEDGMENTS}

The authors wish to thank the Swiss National Science Foundation (grant IZK0Z2-141692) which funded the visit of S. Rosset to the Biomimetics lab.

\section{REFERENCES}

1. A. Wingert, M. Lichter, S. Dubowsky, and M. Hafez, "Hyper-redundant robot manipulators actuated by optimized binary dielectric polymers," in Proceedings of SPIE - The International Society for Optical Engineering, 4695, pp. 415-423, 2002.

2. V. Sujan and S. Dubowsky, "Design of a lightweight hyper-redundant deployable binary manipulator," Journal of Mechanical Design 126(1), pp. 29-39, 2004.

3. G. Kovacs, P. Lochmatter, and M. Wissler, "An arm wrestling robot driven by dielectric elastomer actuators," Smart Materials and Structures 16(2), pp. s306-s317, 2007.

4. J. J. Loverich, I. Kanno, and H. Kotera, "Concepts for a new class of all-polymer micropumps," Lab on a Chip - Miniaturisation for Chemistry and Biology 6(9), pp. 1147-1154, 2006.

5. M. Matysek, P. Lotz, K. Flittner, and H. F. Schlaak, "Vibrotactile display for mobile applications based on dielectric elastomer stack actuators," in Proceedings of SPIE - The International Society for Optical Engineering, Y. Bar-Cohen, ed., 7642, p. 76420D, SPIE, 2010.

6. S. Akbari and H. R. Shea, "An array of 100um x 100um dielectric elastomer actuators with $80 \%$ strain for tissue engineering applications," Sensors and Actuators A: Physical 186, pp. 236 - 241, 2012.

7. M. Aschwanden and A. Stemmer, "Low voltage, highly tunable diffraction grating based on dielectric elastomer actuators," in Proceedings of SPIE - The International Society for Optical Engineering, 6524, pp. 65241N-10, SPIE, (San Diego, California, USA), 2007.

8. F. Carpi, G. Frediani, S. Turco, and D. De Rossi, "Bioinspired tunable lens with muscle-like electroactive elastomers," Advanced Functional Materials 21(21), pp. 4152-4158, 2011. 
9. M. Kollosche, S. Döring, G. Kofod, and J. Stumpe, "A novel approach to tunable diffractive transmission gratings based on dielectric elastomer actuators," in Proceedings of SPIE - The International Society for Optical Engineering, Y. Bar-Cohen, ed., 7642(1), p. 76422Y, SPIE, 2010.

10. M. Tormen, Y.-A. Peter, P. Niedermann, A. Hoogerwerf, and R. Stanley, "Deformable mems grating for wide tunability and high operating speed," Journal of Optics A: Pure and Applied Optics 8(7), p. S337, 2006.

11. C. W. Wong, Y. Jeon, G. Barbastathis, and S.-G. Kim, "Analog tunable gratings driven by thin-film piezoelectric microelectromechanical actuators," Appl. Opt. 42, pp. 621-626, Feb 2003.

12. S. Rosset, P. Gebbers, B. M. O'Brien, and H. R. Shea, "The need for speed," in Proceedings of SPIE - The International Society for Optical Engineering, 8340, pp. 834004-834004-12, 2012.

13. B. O'Brien, J. Thode, I. Anderson, E. Calius, E. Haemmerle, and S. Xie, "Integrated extension sensor based on resistance and voltage measurement for a dielectric elastomer," in Proceedings of SPIE - The International Society for Optical Engineering, 6524, pp. 652415-11, SPIE, 2007.

14. T. Gisby, S. Xie, E. Calius, and I. Anderson, "Integrated sensing and actuation of muscle-like actuators," in Proceedings of SPIE - The International Society for Optical Engineering, Proceedings of SPIE - The International Society for Optical Engineering 7287, pp. 728707-1, 2009.

15. T. A. Gisby, B. M. O'Brien, S. Q. Xie, E. P. Calius, and I. A. Anderson, "Closed loop control of dielectric elastomer actuators," in Proceedings of SPIE - The International Society for Optical Engineering, 7976, pp. 797620-797620-9, 2011.

16. T. A. Gisby, B. M. O'Brien, and I. A. Anderson, "How far and how hard: tactile feedback for robotic manipulators," in Proceedings of SPIE - The International Society for Optical Engineering, Y. Bar-Cohen, ed., 8340, p. 83401S, SPIE, 2012.

17. T. Mckay, E. Calius, and I. Anderson, "The dielectric constant of $3 \mathrm{~m}$ vhb: A parameter in dispute," in Proceedings of SPIE - The International Society for Optical Engineering, 7287, p. 72870P, 2009. 